\title{
Endogenous Versus Exogenous Shocks in Complex Networks: an Empirical Test Using Book Sale Ranking
}

\author{
D. Sornette, ${ }^{1,2}$ F. Deschâtres,${ }^{3}$ T. Gilbert,${ }^{4}$ and Y. Ageon ${ }^{2}$ \\ ${ }^{1}$ Institute of Geophysics and Planetary Physics and Department of Earth and Space Sciences, \\ University of California, Los Angeles, CA 90095 \\ ${ }^{2}$ Laboratoire de Physique de la Matière Condensée, \\ CNRS UMR 6622 and Université de Nice-Sophia Antipolis, 06108 Nice Cedex 2, France \\ ${ }^{3}$ Ecole Normale Supérieure, rue d'Ulm, Paris, France \\ ${ }^{4}$ Haas School of Business, University of California, Berkeley, California 94720, USA
}

(Dated: October 24, 2018)

\begin{abstract}
We study the precursory and recovery signatures accompanying shocks in complex networks, that we test on a unique database of the amazon.com ranking of book sales. We find clear distinguishing signatures classifying two types of sales peaks. Exogenous peaks occur abruptly and are followed by a power law relaxation, while endogenous peaks occur after a progressively accelerating power law growth followed by an approximately symmetrical power law relaxation which is slower than for exogenous peaks. These results are rationalized quantitatively by a simple model of epidemic propagation of interactions with long memory within a network of acquaintances. The observed relaxation of sales implies that the sales dynamics is dominated by cascades rather than by the direct effects of news or advertisements, indicating that the social network is close to critical.
\end{abstract}

PACS numbers: 64.60.Ak; 02.50.Ey; 91.30.Dk

A fundamental question in the theory of out-ofequilibrium systems is whether the response function to external kicks can be related to spontaneous internal fluctuations [1]. At equilibrium, this is solved by the fluctuation-dissipation theorem connecting susceptibility and noise. In many complex systems, this question amounts to distinguishing between endogeneity and exogeneity and is important for understanding the relative effects of self-organization versus external impacts. This is difficult in most physical systems because externally imposed perturbations may lie outside the complex attractor which itself may exhibit bifurcations. Therefore, observable perturbations are often misclassified. For this reason, we have studied a non-physical system in which the dividing line between endogenous and exogenous shocks is clear in the hope that it will lead to insights about complex physical systems. We study the dynamics of commercial growth and its relaxation in the social system of interacting buyers, obtained from an Amazon.com database of book sales. We do see a characteristic difference in behavior between endogenous and exogenous shocks.

Every book that has sold at least one copy on the online retailer Amazon is automatically assigned a sales rank. Typically, two (respectively ten) sales a day puts a title in the top 10,000 (respectively 1,000) sellers. The top 100 (respectively 10) sell more than about 30 (respectively 100) books per day through Amazon. Its American website, Amazon.com, updates the ranks of its top 10,000 books every hour, according to a formula accounting for recent sales and the entire sales history of the book. Direct sales are confidential data but their statistical properties can be reconstructed approximately by careful ob-

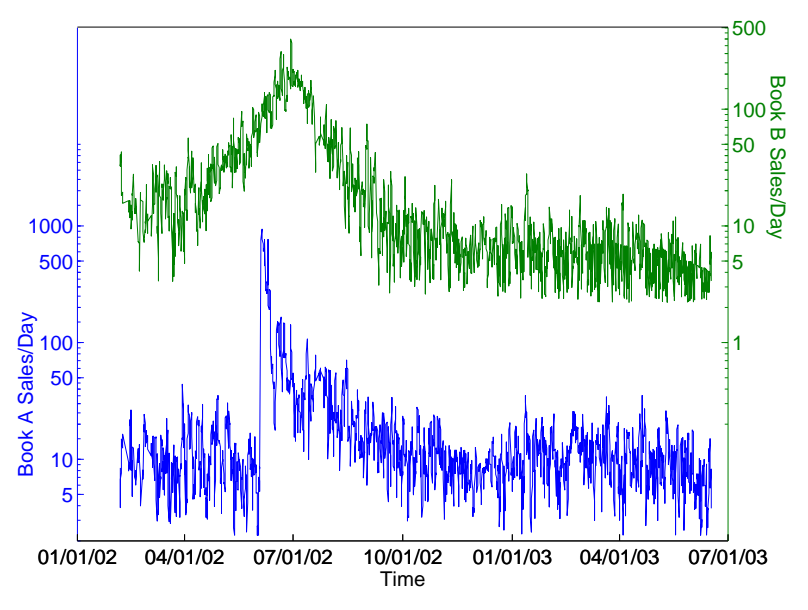

FIG. 1: Time evolution over a year and a half of the sales per day of two books: Book A (bottom, left scale) is "Strong Women Stay Young" by Dr. M. Nelson and Book B (top, right scale) is "Heaven and Earth (Three Sisters Island Trilogy)" by N. Roberts.

servations 2]. The complementary cumulative distribution $P(s)$ of sales $s$ can be approximated by a stationary power law $P(s)=C / s^{\mu}$ with $\mu \approx 2$ in the range of sales from a few books sold per day to a few hundreds (see figure in [2]). We use this power law to transform book ranks $r(s)=N P(s)$ into sales $s$ according to the formula $s=(N C / r)^{1 / \mu}$, where $N$ is the total number of books used to normalize the distribution. Thus, a time series of the rank $r$ of a given book as a function of time, sampled at a given rate, can be transformed into a time series of instantaneous sales flux, through this conversion. 
The time series of ranks of thousands of books have been recorded with a six-hour sampling rate by JungleScan (http://www.junglescan.com). From the hundreds of books with at least one year of recording, we have selected all that have reached the top 50 in Amazon sales rank. We qualify a peak in sales as a local maximum over a three-month time window which is at least $k=2.5$ times larger than the average of the time series over the three months. In addition, we request that there is at least 15 days of data after each peak and 4 days before. Out of some 14,000 books on Junglescan on April 2004, our algorithm detects about 1000 such peaks. Fig. 11 shows about 1.5 years of data for two books, which are illustrative of the two classes found in this study. Book A jumped on June 5, 2002, from rank in the 2,000s to rank 6 in less than 12 hours. On June 4, 2002, the New York Times published an article crediting the "groundbreaking research done by Dr. Miriam Nelson" and advising the female reader, interested in having a youthful postmenopausal body, to buy the book and consult it directly [3]. This case is the archetype of an "exogenous" shock.

In contrast, Book B culminated at the end of June 2002 after a slow and continuous growth, with no such newspaper article, followed by a similar almost symmetrical decay, the entire process taking about 4 months. The peak for Book B belongs to the class of endogenous shocks as described below. Qualitatively, such endogenous growth is well explained in Ref. [4] by taking the example of the book "Divine Secrets of the Ya-Ya Sisterhood" by R. Wells, which became a bestseller two years after publication, with no major advertising campaign. Following the reading of this originally small budget book, "Women began forming $Y a-Y a$ Sisterhood groups of their own [...]. The word about $Y a-Y a$ was spreading [...] from reading group to reading group, from $Y a-Y a$ group to $Y a-Y a$ group" [4].

Such social epidemic process can be captured by the following simple model. The instantaneous sales flux of a given book results from a combination of external forces such as news, advertisement, selling campaign, and of social influences in which each past reader may impregnate other potential readers in her network of acquaintances with the desire to buy the book. This impact of a reader onto other readers is not instantaneous as people react at a variety of time scales. This latency can be described by a memory kernel $\phi\left(t-t_{i}\right)$ giving the probability that a buy at time $t_{i}$ leads to another buy at a later time $t$ by another person in direct contact with the first buyer. Starting from an initial buyer (the "mother" buyer) who notices the book (either from exogenous news or by chance), she may trigger buying by first-generation "daughters," who themselves propagate the buying drive to their own friends, who become second-generation buyers, and so on. We describe the sum of all buys by a conditional Poisson branching process with intensity

$$
\lambda(t)=S(t)+\sum_{i \mid t_{i} \leq t} \mu_{i} \phi\left(t-t_{i}\right)
$$

where $\mu_{i}$ is number of potential buyers influenced by the buyer $i$ who bought earlier at time $t_{i}$. $S(t)$ is the rate of sales initiated spontaneously without influence from other previous buyers; it can be decomposed into the sum of a white-noise process with power law distribution representing small triggering factors (which contribute to the endogenous shocks) and a jump process (Dirac distributions) modeling massive media coverage and advertisement campaigns for instance (exogenous shocks). We note that the distinction between endogenous and exogenous is in general murky and cannot be decided with $100 \%$ certainty for most books; the correct approach is probabilistic and relies on the analysis of an ensemble of cases, as presented below.

Taking the ensemble average of (11) gives the selfconsistent mean-field equation

$$
s(t)=\langle\lambda(t)\rangle=S(t)+n \int_{-\infty}^{t} d \tau \phi(t-\tau) s(\tau),
$$

where $n=\langle\mu\rangle$ is the average number of buys of first generation triggered by any "mother" within her acquaintance network (also called the branching ratio) and depends on the network topology as well as on the social behavior of influences. The Green function $K(t)$ of (2) corresponding to $S(t)=\delta(t)$ (exogenous shock) is easily obtained by taking its Laplace transform and corresponds to the exogenous response function. We postulate that the "bare propagator" is of the form $\phi\left(t-t_{i}\right) \sim 1 / t^{1+\theta}$ with $0<\theta<1$ corresponding to a long-memory process that are commonly observed for sale relaxations. Then,

$$
s_{\text {exo }} \equiv K(t) \sim 1 /\left(t-t_{c}\right)^{1-\theta},
$$

for $t<t^{*} \propto 1 /(1-n)^{1 / \theta}$ and $K(t) \sim 1 / t^{1+\theta}$ for $t>t^{*}$ and $n<1$. Expression (2) can then be written $s(t)=\int_{-\infty}^{t} d \tau K(t-\tau) S(\tau)$. Close to the critical point $n \approx 1$, the cascade of generations embodied in (2) renormalizes the memory kernel $\phi\left(t-t_{i}\right)$ into a dressed or renormalized memory kernel $K\left(t-t_{i}\right)$ [5], giving the probability that a buy at time $t_{i}$ leads to another buy by another person at a later time $t$ through any possible generation lineage $\left(\int_{0}^{\infty} K(t) d t=n /(1-n)\right.$ is the average number of buys triggered by one buy). Thus, if we interpret the sharp peak of Book A observed in Fig. 1 as the impact on the social network of women created by the extraordinarily favorable appraisal of the New York Times, the decay of the sales flow that followed gives a direct measure of its response function $K(t)$ : we find indeed a power law (not shown) as predicted by (3) with $\theta=0.3 \pm 0.1$. Such power law dependence of the relaxation rate of book sales on Amazon.com is the hallmark 
of a long-memory process characterizing the dynamics of influences within the complex social network. These laws are similar to the relaxation of seismic activity after an earthquake, known as Omori's law 6] and has also been found in the response rates of internauts to an exogenous shock, such as the publication of the URL in a newspaper interview 7], and in the relaxation of volatility shocks in the stock market [8].

In the absence of strong external influences, a peak in book sales can occur spontaneously due to the interplay between a continuous stochastic flow of small external news and the amplifying impact of the epidemic cascade of social influences. We propose that this mechanism explains the sales time series of books such as Book B shown in Fig. 11 Technically, the problem amounts to calculating the average sales trajectory before and after a peak, conditioned on the existence of a peak. We use the standard result for stochastic processes $X(t)$ with finite variance and covariance that $\left\langle X(t) \mid X(t=0)=X_{0}\right\rangle \propto$ $\operatorname{Cov}\left(X(t), X_{0}\right)$. Applying this result to $\lambda(t)$ defined in (11) gives $\operatorname{Cov}\left(\lambda(t), \lambda_{0}\right) \propto \int_{-\infty}^{t} d \tau K(t-\tau) K(-\tau)$. This expression gives the average growth of the sales before such an "endogenous" peak and the relaxation after the peak, which is proportional to

$$
s_{\text {endo }}(t) \sim 1 /\left|t-t_{c}\right|^{1-2 \theta},
$$

for $K(t)$ given by (3) 5]. The prediction that the relaxation following an exogenous shock should happen faster (3) (larger exponent $1-\theta$ ) than (4) for an endogenous shock (with exponent $1-2 \theta$ ) reflects the fact that an endogenous shock results from a precursory process that inpregnates the network much more over a longer time and thus has a longer lived influence. The prediction (4) is verified for Book $B$ with good precision (not shown) with the same $\theta=0.3 \pm 0.1$.

Among the thousand peaks in our database, many are followed by complicated trajectories probably due to multiple external influences. Nevertheless, we fitted all sales time dependence after each of the thousand peaks by a power law of the type (3) or (4) and selected for further analysis those that give a correlation coefficient $R$ better than 0.95 . This leaves us with 138 peaks. This criterion ensures that we extract clear response functions among the generally complex time series. Our purpose is not so much to show that the response function is a power law but that, if it is, then we classify another class of so-called "endogenous" peaks with precursory and decay rates that can be predicted. Our results below are not changed qualitatively by changing this threshold on $R$ in the range from 0.8 to close to 1 . We also find the good feature that the classification discussed below in terms of exogenous versus endogenous peaks improves (fewer misclassifications) as the threshold increases, conforting the hypothesis that the response function of a book sales to a strong advertisement is of the form (3). Our goal is thus

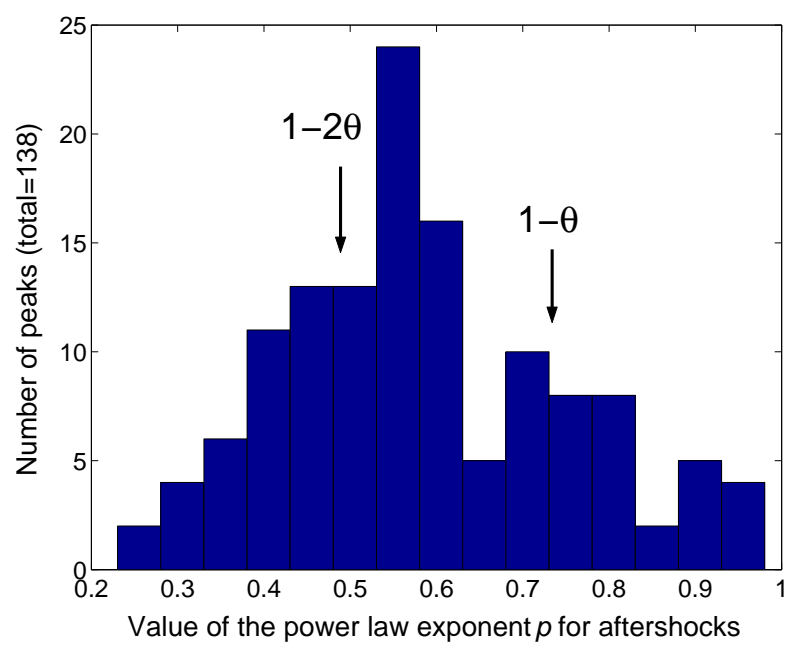

FIG. 2: Histogram of the exponents $p$ of the fits of book sales as a function of time by the power law $\sim 1 /\left(t-t_{c}\right)^{p}$. The arrows indicate the values $p=1-\theta$ (expected for exogenous peaks) and $p=1-2 \theta$ (for endogenous peaks), with $\theta \approx 0.3$ (see text).

to extract the pure cases and show that they are consistent with the model, which has rigid predictions linking the exponents of the precursory and relaxation behavior of exogenous and endogenous cases. In turn, this should permit to use the general formulation (1) with an arbitrary time-dependent source term to describe general situations.

For each of the 138 selected peaks, we measure the exponent $p$ characterizing the power laws $1 /\left(t-t_{c}\right)^{p}$ describing the relaxation of the sales after each peak. We perform a mean-square fit from the peak up to a time $t_{\text {end }}$, where $t_{\text {end }}$ is varied from 15 days to the first mininum of the sales between 25 days and 6 months after the peak. Once all fits are performed for these different time windows, we select the window with the highest correlation coefficient of the fit to the data (we also used other criteria such as selecting the window with the smaller exponent, without altering the results significantly). The histogram of $p$-values shown in Fig. 2 exhibits two distinct clusters, one with a median at $p \approx 0.75$ and the other with a median at $p \approx 0.45$, compatible with the predictions (3) and (4) with the choice $\theta=0.3 \pm 0.1$. This classifies the first (respectively second) cluster as exogenous (respectively endogenous). This histogram is robust with respect to variations in our procedure, such as different windows and peak thresholds.

According to our model, the peaks belonging to the cluster with high $p(p \approx 0.7)$ should be in the exogenous class, and therefore should be reached by abrupt jumps without detectable precursory growth. Conversely, the peaks belonging to the cluster with $p \approx 0.4$ should be in the endogenous class, and therefore should be asso- 
ciated with a progressive power law precursory growth $1 /\left(t_{c}-t\right)^{p}$ with exponent $p=1-2 \theta$. To check this prediction, the following algorithm categorizes the growth of sales before each of the peaks according to its acceleration pattern. We differentiate between peaks which have an increase in sales in a four day period by a factor of at least $k_{\text {exo }}$ prior to the peak and peaks that have an increase in sales by a factor of less than $k_{\text {endo }}$. We find that the larger $k_{\text {exo }}$ is, the larger is the exponent $p$ of the average relaxation for books that have an increase in sales by a factor more than $k_{\text {exo }}$. Conversely, the smaller $k_{\text {endo }}$ is, the smaller is the exponent $p$ of the average relaxation for books that have an increase in sales by a factor less than $k_{\text {exo }}$. These results confirm the predictions of the model. Quantitatively, we first apply a stringent selection with $k_{\text {exo }}=30, k_{\text {endo }}=2$. This implies that peaks, for which the acceleration factor is between 2 and 30, are disgarded in order to get clean signals. Out of the 138 peaks, this leaves us with 30 peaks. We then average the precursory and relaxation behavior of sales for the class of peaks classified as endogenous with $k_{\text {endo }}=2$ and as exogenous with $k_{\text {exo }}=30$. Fig. 3] confirms nicely the existence of two classes, with a symmetric precursory and relaxation behavior for endogenous peaks, and with all three power laws accounted for by a single value $\theta=0.3 \pm 0.1$. The two clusters remain significant for less restrictive $k_{\text {exo }}$ and $k_{\text {endo }}$. While the theoretical predictions have been derived for ensemble averages, we find that more than $80 \%$ among the 138 peaks we have analyzed with at least a year of data and that reached the top 50 among all books also obey $1 /\left(t-t_{c}\right)^{1-\theta}$ or $1 /\left(t-t_{c}\right)^{1-2 \theta}$ individually with reasonable precision. Our finding that the classifications of endogenous versus exogenous match so well on individual books is extremely significant with only 1 chance in about $10^{8}$ that this result could be obtained by chance only.

The values of the exponents smaller than one (close to $1-\theta$ and $1-2 \theta$ ) both for exogenous and endogenous relaxations imply that the sales dynamics is dominated by cascades involving high-order generations rather than by interactions stopping after first-generation buy triggering. Indeed, if buys were initiated mostly by the direct effects of news or advertisements, and not much by triggering cascades in the acquaintance network, the cascade model predicts that we should then measure an exponent $1+\theta$ given by the "bare" memory kernel $\phi(t)$. This implies that the average number $n$ of impregnated buyers per initial buyer in the social epidemic model is on average very close to the critical value 1 , because the renormalization from $\phi(t)$ to $K(t)$ given by (3) only operates close to criticality characterized by the occurrence of large cascades of buys. This offers a new signature of criticality in self-organized networks 9].

Extreme events in complex physical systems, particularly those which seem to involve self-organized criticality, are often viewed as having an endogenous source [10].

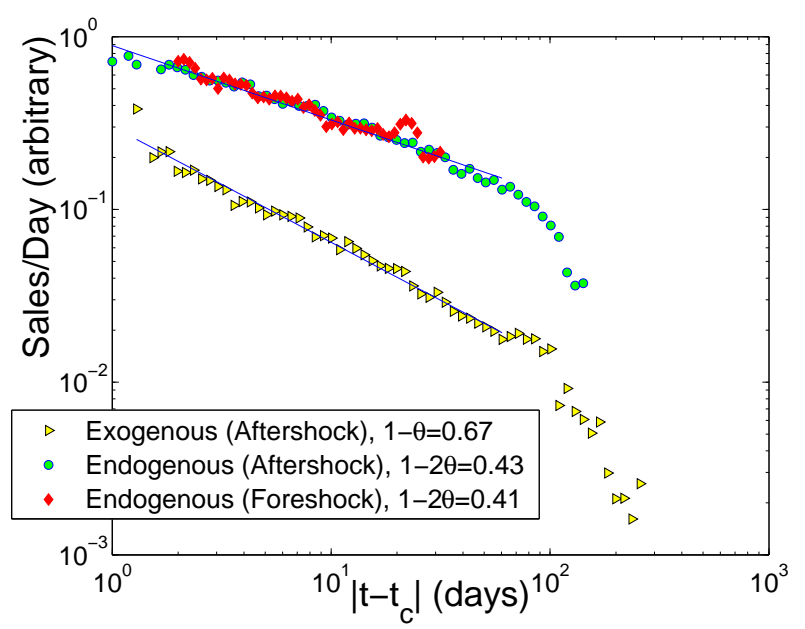

FIG. 3: Precursory ("foreshock") and relaxation ("aftershock") of sales around peaks obtained after averaging over books in each class classified according to the precursory acceleration (see text).

Our work shows that the issue is not clear-cut as endogenous and exogenous shocks may lead to similar power law signatures, which can however be distinguished by a careful classification. This offers new ideas for probing selforganized critical systems as done recently for the OlamiFeder-Christensen sandpile model 11]. We also note that the distinction between jammed states (constructed by fast processes) 12 versus fragile states (formed by slow and delicate accumulation of perturbations) 13 of granular media and other "soft-matter" systems is based in part on the nature of their preparation and on their response to finite and short-lived perturbations versus infinitesimal continuously repeated ones. Recognizing the importance of the nature of the perturbation as suggested here could provide new insights in the organization of granular media and new experimental questions, such as new ways of analyzing the history. Similar considerations apply to memory retrieval using hysteresis loops in magnets 14]. More generally, physical systems with many competing equilibria such as glasses and spinglasses are known to betray their history-dependent organization differently depending on whether they are subjected to large non-local perturbations versus continuous slow forcing [15]. Our classification of endogenous versus exogenous shocks presented here should encourage researchers to analyze complex physical systems similarly.

Acknowledgments: We thank W.-X. Zhou and A. Helmstetter for exchanges. This work was performed in part while FD and TG were at the Department of Earth and Space Sciences at UCLA as well as FD at the University of Nice. 
[1] Ruelle, D., Phys. Today 57(5), 48 (2004).

[2] Rosenthal, M., http://www.fonerbooks.com/surfing.htm (2004).

[3] Brody, J., The New York Times F 7 (June 4, 2002).

[4] Gladwell, M. The tipping point (Back Bay Books, 2002).

[5] Sornette, D. \& Helmstetter, A. Physica A 318, 577-591 (2003).

[6] Omori, F. J. Coll. Sci. Imp. Uni. 7, 111 (1894).

[7] Johansen, A. \& Sornette, D. Physica A 276, 338-345 (2000); Johansen, A. Physica A 296, 539-546 (2001).
[8] Sornette et al., Risk 16, 67 (2003).

[9] A. Barabási, Linked (Perseus, Cambridge, 2002).

[10] Bak, P. (1996) How Nature Works (Copernicus, New York).

[11] Helmstetter, A. et al. (cond-mat/0312689)

[12] Edwards, S.F. and D.V. Grinev, Adv. Phys. 51, 1669 (2002).

[13] Cates, M.E. et al., Phys. Rev. Lett. 81, 1841 (1998).

[14] Perkovic. O. and J. P. Sethna, J. Appl. Phys. 81, 1590 (1997).

[15] Lamarcq, J. et al., Phys. Rev. B. 68, 012404 (2003). 